$\underline{\text { Preprint typeset in JHEP style - HYPER VERSION }}$

\title{
Probing spacetime noncommutative constant via charged astrophysical black hole lensing
}

\author{
Chikun Ding * \\ Department of Physics and Information Engineering, \\ Hunan Institute of Humanities, Science and Technology, \\ Loudi, Hunan 417000, P. R. China \\ Jiliang Jing ${ }^{\dagger}$ \\ Department of Physics, and Key Laboratory of Low Dimensional Quantum Structures \\ and Quantum Control of Ministry of Education, Hunan Normal University, \\ Changsha, Hunan 410081, P. R. China
}

\begin{abstract}
:
We study the influence of the spacetime noncommutative parameter on the strong field gravitational lensing in the noncommutative Reissner-Nordström black-hole spacetime. Supposing that the gravitational field of the supermassive central object of the Galaxy is described by this metric, we estimate the numerical values of the coefficients and observables for strong gravitational lensing. Our results show that with the increase of the parameter $\sqrt{\vartheta}$, the observables $\theta_{\infty}$ and $r_{m}$ decrease, while $s$ increases. Our results also show that i) if $\sqrt{\vartheta}$ is strong, the observables are close to those of the noncommutative Schwarzschild black hole lensing; ii) if $\sqrt{\vartheta}$ is weak, the observables are close to those of the commutative Reissner-Nordström black hole lensing; iii) the detectable scope of $\vartheta$ in a noncommutative Reissner-Nordström black hole lensing is $0.12 \leq \sqrt{\vartheta} \leq 0.26$, which is wider than that in a noncommutative Schwarzschild black hole lensing, $0.18 \leq \sqrt{\vartheta} \leq 0.26$. This may offer a way to probe the spacetime noncommutative constant $\vartheta$ by the astronomical instruments in the future.
\end{abstract}

Keywords: Black Holes, Gravitational Lensing, Noncommutative Spacetime.

\footnotetext{
${ }^{*}$ Email: dingchikun@163.com, QQ: 649433259

†Email: jljing@hunnu.edu.cn
} 


\section{Contents}

1. Introduction 1

2. The charged astrophysical black hole 3

3. Deflection angle in the charged noncommutative black hole spacetime 4

4. Numerical estimation of observational gravitational lensing parameters 10

5. Summary

\section{Introduction}

Quantum mechanics teach us that the emergence of a minimal length is a natural requirement when quantum features of phase space are considered. It also holds true to spacetime [1]. The presence of a minimal length implies that singularities in general relativity and ultraviolet divergences in quantum field theory are nothing but spurious effects due to the inadequacy of the formalism at small scales/extreme energies, rather than actual physical phenomena. Given this background, noncommutative geometry have been devoted to implementing a minimal length in physical theories and curing the aforementioned pathologies. In the string theory, coordinates of the target spacetime become noncommutating operators on a $D$-brane as [2]

$$
\left[\hat{x}^{\mu}, \hat{x}^{\nu}\right]=i \vartheta^{\mu \nu}
$$

where $\vartheta^{\mu \nu}$ is a real, anti-symmetric and constant tensor which determines the fundamental cell discretization of spacetime much in the same way as the Planck constant $\hbar$ discretizes the phase space, $\left[\hat{x}_{i}, \hat{p}_{j}\right]=i \hbar \delta_{i j}$. Motivated by string theory arguments, noncommutative spacetime has been reconsidered again and is believed to afford a starting point to quantum gravity.

Noncommutative spacetime is not a new conception, and coordinate noncommutativity also appears in another fields, such as in quantum Hall effect [3], the noncommutative Landau problem [4], cosmology [5], the model of a very slowly moving charged particle on a constant magnetic field [6], a strong magnetic field [7], the Chern-Simon's theory [8], and so on. The idea of noncommutative spacetime dates back to Snyder [9] who used the noncommutative structure of spacetime to introduce a small length scale cut-off in field theory without breaking Lorentz invariance and Yang [10] who extended Snyder's work to quantize spacetime in 1947 before the renormalization theory. Noncommutative geometry [11] is a branch of mathematics that has many applications in physics, a good review of the noncommutative spacetime is in [12, 13].

The fundamental notion of the noncommutative geometry is that the picture of spacetime as a manifold of points breaks down at distance scales of the order of the Planck length: Spacetime events cannot be localized with an accuracy given by Planck length [13] as well as particles do in the quantum phase space. So that the points on the classical commutative manifold should then be 
replaced by states on a noncommutative algebra and the point-like object is replaced by a smeared object [14] to cure the singularity problems at the terminal stage of black hole evaporation [15].

The approach to noncommutative quantum field theory follows two paths: one is based on the Weyl-Wigner- Moyal *-product and the other on coordinate coherent state formalism [14]. In a recent paper [16], following the coherent state approach, it has been shown that Lorentz invariance and unitary, which are controversial questions raised in the *-product approach, can be achieved by assuming

$$
\vartheta^{\mu \nu}=\vartheta \operatorname{diag}\left(\epsilon_{1}, \ldots, \epsilon_{D / 2}\right)
$$

where $\vartheta \sqrt{17}$ is a constant which has the dimension of lengt $h^{2}, D$ is the dimension of spacetime [18] and, there isn't any UV/IR mixing. Inspire by these results, various black hole solutions of noncommutative spacetime have been found [19]; thermodynamic properties of the noncommutative black hole were studied in [20]; the evaporation of the noncommutative black hole was studied in [21]; quantum tunneling of noncommutative Kerr black hole was studied in [22]; quantized entropy was studied in [23], and so on.

Noncommutative black holes are currently the richest class of quantum gravity black holes [24] which are connected with a recently proposed ultraviolet complete quantum gravity [25] and, have been recently taken into account in Monte Carlo simulations as reliable candidate models to describe the conjectured production of microscopic black holes in particle accelerators [26]. It is interesting that the noncommutative spacetime coordinates introduce a new fundamental natural length scale

$$
l_{N C}=\sqrt{\vartheta}
$$

the effects of noncommutativity are increasing important from main-sequence stars to neutron stars and it might have a relevant impact on black-hole physics [27]. In this paper we will continue to study its influence on strong gravitational lensing.

In previous work [28], we supposed that the gravitational field of the supermassive central object of the Galaxy can be described by the noncommutative Schwarzschild metric, and estimated the numerical values of the coefficients and observables for strong gravitational lensing. In comparison to the commutative Reissner-Nordström black hole, we find that the influences of the spacetime noncommutative parameter is similar to those of the charge, but these influences are much smaller. However some problems would appear: (i) if the spacetime noncommutative constant $\vartheta$ is very strong or the charge quantity of the black hole is very small, it is difficult to distinguish the influences of the $\vartheta$ from the charge; (ii) if the spacetime noncommutative constant $\vartheta$ is very weak, the study of the noncommutative Schwarzschild lensing cannot probe it at all since that its effects cannot obviously deviate from those of the commutative Schwarzschild lensing until $\sqrt{\vartheta} \geq 0.18$. Therefore it is not enough to study the lensing in the noncommutative Schwarzschild black-hole spacetime. Hence in this paper, we plan to study the influence of this constant on strong gravitational lensing in the noncommutative Reissner-Nordström black-hole spacetime.

Gravitational lensing continues to be a major source of insight into gravitation and cosmology [29]. When the lens is a black hole, a strong field treatment of gravitational lensing [30, 31, 32, 33, [34, 35] is needed. The relativistic images of strong gravitational lensing could provide a profound verification of alternative theories of gravity. Thus, the study of the strong gravitational lensing becomes appealing recent years. Bhadra et al [36] [37] have considered the Gibbons-Maeda-GarfinkleHorowitz-Strominger black hole lensing. Eiroa et al [38] have studied the Reissner-Nordström black hole lensing. Konoplya [39] has studied the corrections to the deflection angle and time delay 
of black hole lensing immersed in a uniform magnetic field. Majumdar [40] has investigated the dilaton-de Sitter black hole lensing. Perlick 41 has obtained an exact lens equation and used it to study Barriola-Vilenkin monopole black hole lensing. Bin-Nun [42] studied Sagittarius A* (Sgr $\mathrm{A}^{*}$ ) lensing; Wei et al studied the strong gravitational lensing in Kerr-Taub-NUT spacetime [43], braneworld black holes lensing were studied in [44, and so on.

The plan of our paper is organized as follows. In Sec. II we study some properties of the noncommutative Reissner-Nordström black hole metric. In Sec. III we adopt Bozza's method and obtain the deflection angles for light rays propagating in the noncommutative Reissner-Nordström black hole spacetime. In Sec. IV we suppose that the gravitational field of the supermassive black hole at the centre of our Galaxy can be described by this metric and then obtain the numerical results for the observational gravitational lensing parameters defined in Sec. III. Then, we make a comparison among the properties of gravitational lensing in the noncommutative/commutative Reissner-Nordström and noncommutative Schwarzschild metrics. In Sec. V, we present a summary.

\section{The charged astrophysical black hole}

The Reissner-Nordström solution is a natural result of the Einstein-Maxwell field equation which shows that a black hole can possess some net charge. But there exists a general consensus that astrophysical objects with large amounts of charge can not exist in nature since that astrophysical objects are always surrounded by some plasma, which is a very good conductor 445. The same reasoning is applied to black holes [45] which shows that the real astrophysical black holes should be neutral. This point of view had been challenged by several researchers [46]. Recently, Some authors found that the charged black hole can be formed during charged stellar collapse [47] or accreting process of a neutral black bole 48].

Using the brane-world-inspired charge-leaking mechanism, Cuesta et al. 49 provide a natural explanation for the formation of charged black holes after supernova collapse of massive stars. In brane world models free particles localized on the brane can leak out to the extra space. If there were color confinement in the bulk, electrons would be more able to escape than quarks and protons which generates an electric charge asymmetry on the brane matter densities. A tiny charge asymmetry would generate small electromagnetic fields which can be substantially magnified [50] by some astrophysical processes, such as a supernova core collapse. According to Ref. [51, vacuum polarization occurring during the formation of a Reissner-Nordström black hole may generate gamma-ray bursts, provided that the net charge-to-mass ratio, $\xi=Q /(2 M \sqrt{G})$, be of the order 0.005 to 0.5 .

Therefore considering strong gravitational lensing in charged black hole spacetime is practical. The line element of the noncommutative Reissner-Nordström black hole reads [15]

$$
d s^{2}=-f(r) d t^{2}+\frac{d r^{2}}{f(r)}+r^{2}\left(d \theta^{2}+\sin ^{2} \theta d \phi^{2}\right),
$$

and

$$
\begin{aligned}
& f(r)=1-\frac{4 G M}{c^{2} r \sqrt{\pi}} \gamma\left(\frac{3}{2}, \frac{r^{2}}{4 \vartheta}\right)+\frac{G Q^{2}}{\pi c^{4} r^{2}} F(r), \\
& F(r)=\left[\gamma^{2}\left(\frac{1}{2}, \frac{r^{2}}{4 \vartheta}\right)-\frac{r}{\sqrt{2 \vartheta}} \gamma\left(\frac{1}{2}, \frac{r^{2}}{2 \vartheta}\right)+\sqrt{\frac{2}{\vartheta}} r \gamma\left(\frac{3}{2}, \frac{r^{2}}{4 \vartheta}\right)\right],
\end{aligned}
$$


where $\gamma(s, x)$ is the lower incomplete Gamma function:

$$
\gamma(s, x) \equiv \int_{0}^{x} d t t^{s-1} e^{-t}
$$

and $\vartheta$ is a spacetime noncommutative parameter [17], $G$ is Newtonian constant, $c$ is light velocity in vacuum. The commutative Reissner-Nordström metric is obtained from (2.1) in the limit $r / \sqrt{\vartheta} \rightarrow \infty$. Equation (2.1) leads to the mass distribution $m(r)=2 M \gamma\left(3 / 2, r^{2} / 4 \vartheta\right) / \sqrt{\pi}$ and the charge distribution $q(r)=Q \sqrt{F(r) / \pi}$, where $M$ is the total mass of the source and $Q$ is the total charge of the source.

Depending on the values of $Q, \sqrt{\vartheta}$ and $M$, the metric displays different causal structure: existence of two horizons (non-extremal black hole), one horizon (extremal black hole) or no horizons (massive charged droplet ). Due to $f\left(r_{+}\right)=0$ cannot be solved analytically, we list some values of the maximum charge $Q_{\max }$ and the single horizon $r_{+}$in Table t by letting $2 M=G=c=1^{1}$. Table 1 shows that the maximum charge $Q_{\max }$ decreases with the increase of the spacetime non-

Table 1: Numerical values for the radius of the single event horizon in the charged noncommutative black hole spacetime with different $\sqrt{\vartheta}$ and $Q_{\max }$.

\begin{tabular}{|c|c|c|c|c|c|c|c|}
\hline \hline$\sqrt{\vartheta}$ & 0.262475 & 0.26 & 0.24 & 0.22 & 0.20 & 0.18 & 0.16 \\
\hline$Q_{\max }$ & 0 & 0.09441 & 0.26858 & 0.35411 & 0.41081 & 0.44988 & 0.47593 \\
\hline$r_{+}$ & 0.80802 & 0.78803 & 0.74050 & 0.69423 & 0.65005 & 0.60673 & 0.56632 \\
\hline$\sqrt{\vartheta}$ & 0.14 & 0.12 & 0.10 & 0.08 & 0.06 & 0.04 & 0.02 \\
\hline$Q_{\max }$ & 0.49151 & 0.49867 & 0.50000 & 0.50000 & 0.50000 & 0.50000 & 0.50000 \\
\hline$r_{+}$ & 0.53252 & 0.50794 & 0.51189 & 0.50509 & 0.50022 & 0.50000 & 0.50000 \\
\hline \hline
\end{tabular}

commutative parameter $\sqrt{\vartheta}$. It indicates the restriction of the spacetime non-commutativity on the charge of black hole which implies that i) if $\sqrt{\vartheta}$ is strong, its single horizon is close to that of the noncommutative Schwarzschild black hole; ii) if $\sqrt{\vartheta}$ is weak, its single horizon is close to that of the commutative Reissner-Nordström black hole. When $M>1.9 \sqrt{\vartheta}$ and $0 \leq Q<Q_{\text {max }}$, the two horizons (non-extremal black hole) are given by

$$
r_{ \pm}=\frac{2}{\sqrt{\pi}} \gamma\left(3 / 2, r_{ \pm}^{2} / 4 \vartheta\right)+\frac{Q^{2}}{\pi r_{ \pm}} F\left(r_{ \pm}\right)
$$

which is different from the commutative Reissner-Nordström black hole. The line element (2.1) describes the geometry of a noncommutative black hole and should give us useful insights about possible spacetime noncommutative effects on strong gravitational lensing.

\section{Deflection angle in the charged noncommutative black hole spacetime}

As in [33, 34, 53], the deflection angle for the photon coming from infinite can be expressed as

$$
\alpha\left(r_{0}\right)=I\left(r_{0}\right)-\pi
$$

\footnotetext{
${ }^{1}$ The units we used here and hereafter is the total mass of the black hole $2 M$, i.e., $\frac{c^{2} r}{2 G M} \rightarrow r, \frac{Q}{2 M \sqrt{G}} \rightarrow Q, \frac{c^{2} \sqrt{\vartheta}}{2 G M} \rightarrow$ $\sqrt{\vartheta}$.
} 
where $r_{0}$ is the closest approach distance and $I\left(r_{0}\right)$ is [33, 34]

$$
I\left(r_{0}\right)=2 \int_{r_{0}}^{\infty} \frac{\sqrt{B(r)} d r}{\sqrt{C(r)} \sqrt{\frac{C(r) A\left(r_{0}\right)}{C\left(r_{0}\right) A(r)}-1}}
$$

with

$$
A(r)=f(r), \quad B(r)=1 / f(r), \quad C(r)=r^{2} .
$$

It is easy to determine that as parameter $r_{0}$ decreases, the deflection angle increases. At a certain point, the deflection angle will become $2 \pi$; this means that the light ray will make a complete loop around the compact object before reaching the observer. When $r_{0}$ is equal to the radius of the photon sphere, the deflection angle diverges and the photon is captured.

The photon sphere equation is given by [33, 34]

$$
\frac{C^{\prime}(r)}{C(r)}=\frac{A^{\prime}(r)}{A(r)}
$$

which admits at least one positive solution, and then the largest real root of Eq. (3.4) is defined as the radius of the photon sphere. To the noncommutative Reissner-Nordström black hole metric (2.1), the radius of the photon sphere can be given implicitly by

$$
\begin{aligned}
& r_{p s}=\frac{3}{\sqrt{\pi}} \gamma\left(\frac{3}{2}, \frac{r_{p s}^{2}}{4 \vartheta}\right)-\frac{r_{p s}^{3}}{4 \vartheta \sqrt{\pi \vartheta}} e^{-\frac{r_{p s}^{2}}{4 \vartheta}}-\frac{2 Q^{2}}{\pi r_{p s}} F\left(r_{p s}\right)+\frac{Q^{2}}{\pi \sqrt{\vartheta}} G\left(r_{p s}\right), \\
& G\left(r_{p s}\right)=\left[\gamma\left(\frac{1}{2}, \frac{r_{p s}^{2}}{4 \vartheta}\right)-\frac{r_{p s}}{2 \sqrt{\vartheta}} e^{-\frac{r_{p s}^{2}}{4 \vartheta}}+\frac{r_{p s}^{3}}{4 \vartheta \sqrt{2 \vartheta}}\right] e^{-\frac{r_{p s}^{2}}{4 \vartheta}}-\frac{1}{\sqrt{2}}\left[\frac{1}{2} \gamma\left(\frac{1}{2}, \frac{r_{p s}^{2}}{2 \vartheta}\right)-\gamma\left(\frac{3}{2}, \frac{r_{p s}^{2}}{4 \vartheta}\right)\right]
\end{aligned}
$$

which is an implicit function $f\left(r_{p s}, Q, \sqrt{\vartheta}\right)=0$. It cannot be expressed as an explicit function

\begin{tabular}{|c|c|c|c|c|c|c|c|}
\hline$\sqrt{\vartheta} \quad Q$ & 0.0 & 0.09 & 0.26 & 0.35 & 0.4 & 0.45 & 0.49 \\
\hline 0.0 & 1.50000 & 1.48912 & 1.40368 & 1.31347 & 1.24244 & 1.14686 & 1.03688 \\
\hline 0.14 & 1.50000 & 1.48912 & 1.40368 & 1.31347 & 1.24244 & 1.14686 & 1.03684 \\
\hline 0.16 & 1.50000 & 1.48912 & 1.40368 & 1.31347 & 1.24243 & 1.14676 & - \\
\hline 0.18 & 1.50000 & 1.48912 & 1.40367 & 1.31339 & 1.24216 & 1.14557 & - \\
\hline 0.20 & 1.49995 & 1.48906 & 1.40345 & 1.31263 & 1.24024 & - & - \\
\hline 0.22 & 1.49954 & 1.48859 & 1.40215 & 1.30898 & - & - & - \\
\hline 0.24 & 1.49764 & 1.48647 & 1.39714 & - & - & - & - \\
\hline 0.26 & 1.49151 & 1.47971 & - & - & - & - & - \\
\hline
\end{tabular}
$r_{p s}=g(Q, \sqrt{\vartheta})$, so we list some values of the photon sphere radius in the following table.

Table 2: Numerical values for the radius of the photon sphere in the charged noncommutative black hole spacetime with different $\sqrt{\vartheta}$ and $Q$. For a certain $\sqrt{\vartheta}$ the charge should satisfy the condition $0 \leq Q<Q_{\max }$, so that corresponding slots are left vacant.

From Table 2 we can see that, when $\sqrt{\vartheta} \rightarrow 0$, the photon sphere radius can recover that in the commutative Reissner-Nordström black hole spacetime, i.e. $r_{p s}=\left(3+\sqrt{9-32 Q^{2}}\right) / 4$. The presence 
of $\sqrt{\vartheta}$ decreases the photon sphere radius as well as the charge does. If $\sqrt{\vartheta}$ is weak, the photon sphere radius is close to that of the commutative Reissner-Nordström black-hole lens; if $\sqrt{\vartheta}$ is strong, it is close to that of the noncommutative Schwarzschild black-hole lens. All these features imply that there exist some distinct effects of the noncommutative parameter $\vartheta$ on gravitational lensing in the strong field limit.

Following the method developed by Bozza [53, 54], we define a variable

$$
z=1-\frac{r_{0}}{r}
$$

and obtain

$$
I\left(r_{0}\right)=\int_{0}^{1} R\left(z, r_{0}\right) f\left(z, r_{0}\right) d z
$$

where

$$
R\left(z, r_{0}\right)=\frac{2 r_{0} \sqrt{A(r) B(r) C\left(r_{0}\right)}}{C(r)(1-z)^{2}}=2
$$

and

$$
f\left(z, r_{0}\right)=\frac{1}{\sqrt{A\left(r_{0}\right)-A(r) C\left(r_{0}\right) / C(r)}} .
$$

The function $R\left(z, r_{0}\right)$ is regular for all values of $z$ and $r_{0}$. However, $f\left(z, r_{0}\right)$ diverges as $z$ tends to zero. Thus, we split the integral (3.7) into two parts

$$
\begin{aligned}
& I_{D}\left(r_{0}\right)=\int_{0}^{1} R\left(0, r_{p s}\right) f_{0}\left(z, r_{0}\right) d z \\
& I_{R}\left(r_{0}\right)=\int_{0}^{1}\left[R\left(z, r_{0}\right) f\left(z, r_{0}\right)-R\left(0, r_{p s}\right) f_{0}\left(z, r_{0}\right)\right] d z
\end{aligned}
$$

where $I_{D}\left(r_{0}\right)$ and $I_{R}\left(r_{0}\right)$ denote the divergent and regular parts in the integral (3.7), respectively. To find the order of divergence of the integrand, we expand the argument of the square root in $f\left(z, r_{0}\right)$ to the second order in $z$ and obtain the function $f_{0}\left(z, r_{0}\right)$ :

$$
f_{0}\left(z, r_{0}\right)=\frac{1}{\sqrt{p\left(r_{0}\right) z+q\left(r_{0}\right) z^{2}}}
$$

where

$$
\begin{aligned}
p\left(r_{0}\right)= & 2-\frac{6}{\sqrt{\pi} r_{0}} \gamma\left(\frac{3}{2}, \frac{r_{0}^{2}}{4 \vartheta}\right)+\frac{r_{0}^{2}}{2 \vartheta \sqrt{\pi \vartheta}} e^{-\frac{r_{0}^{2}}{4 \vartheta}}+\frac{4 Q^{2}}{\pi r_{0}^{2}} F\left(r_{0}\right)-\frac{2 Q^{2}}{\pi r_{0} \sqrt{\vartheta}} G\left(r_{0}\right), \\
q\left(r_{0}\right)= & -1+\frac{6}{\sqrt{\pi} r_{0}} \gamma\left(\frac{3}{2}, \frac{r_{0}^{2}}{4 \vartheta}\right)-\frac{r_{0}^{2}}{4 \vartheta \sqrt{\pi \vartheta}} e^{-\frac{r_{0}^{2}}{4 \vartheta}}\left(2+\frac{r_{0}^{2}}{2 \vartheta}\right)-\frac{6 Q^{2}}{\pi r_{0}^{2}} F\left(r_{0}\right) \\
& +\frac{6 Q^{2}}{\pi r_{0} \sqrt{\vartheta}} G\left(r_{0}\right)-\frac{Q^{2} r_{0}^{2}}{2 \pi \vartheta^{2}} H\left(r_{0}\right), \\
H\left(r_{0}\right)= & {\left[-\frac{\sqrt{\vartheta}}{r_{0}} \gamma\left(\frac{1}{2}, \frac{r_{0}^{2}}{4 \vartheta}\right)+e^{-\frac{r_{0}^{2}}{4 \vartheta}}+\sqrt{2}-\frac{r_{0}^{2}}{4 \sqrt{2} \vartheta}\right] e^{-\frac{r_{0}^{2}}{4 \vartheta}} }
\end{aligned}
$$


When $r_{0}$ is equal to the radius of photon sphere $r_{p s}$, the coefficient $p\left(r_{0}\right)$ vanishes and the leading term of the divergence in $f_{0}\left(z, r_{0}\right)$ is $z^{-1}$; thus, the integral (3.7) diverges logarithmically. Close to the divergence, the deflection angle can be expanded in the form

$$
\alpha(\theta)=-\bar{a} \log \left(\frac{\theta D_{O L}}{u_{p s}}-1\right)+\bar{b}+O\left(u-u_{p s}\right),
$$

where

$$
\begin{aligned}
\bar{a}= & \frac{R\left(0, r_{p s}\right)}{2 \sqrt{q\left(r_{p s}\right)}}=\frac{1}{\sqrt{q\left(r_{p s}\right)}}, \\
q\left(r_{p s}\right)= & 1-\frac{r_{p s}^{4}}{8 \vartheta^{2} \sqrt{\pi \vartheta}} e^{-\frac{r_{p s}^{2}}{4 \vartheta}}-\frac{2 Q^{2}}{\pi r_{p s}^{2}} F\left(r_{p s}\right) \\
& -\frac{Q^{2} r_{p s}^{2}}{2 \pi \vartheta^{2}} H\left(r_{p s}\right)+\frac{4 Q^{2}}{\pi r_{p s} \sqrt{\vartheta}} G\left(r_{p s}\right), \\
\bar{b}= & -\pi+b_{R}+\bar{a} \log \frac{4 q^{2}\left(r_{p s}\right)\left[2 A\left(r_{p s}\right)-r_{p s}^{2} A^{\prime \prime}\left(r_{p s}\right)\right]}{p^{\prime 2}\left(r_{p s}\right) u_{p s} r_{p s} \sqrt{A^{3}\left(r_{p s}\right)}} \\
b_{R}= & I_{R}\left(r_{p s}\right), \quad p^{\prime}\left(r_{p s}\right)=\left.\frac{d p}{d r_{0}}\right|_{r_{0}=r_{p s}}, \quad u_{p s}=\frac{r_{p s}}{\sqrt{A\left(r_{p s}\right)}} .
\end{aligned}
$$

$D_{O L}$ denotes the distance between the observer and the gravitational lens, $\bar{a}$ and $\bar{b}$ are the so-called strong field limit coefficients which depend on the metric functions evaluated at $r_{p s}$ and, $u_{p s}$ is the minimum impact parameter. Now we turn to determine $b_{R}$. From Eqs. (3.8 3.12), we obtain

$$
\begin{aligned}
& b_{R}=I_{R}\left(r_{p s}\right)=2 \int_{0}^{1} g(z, \sqrt{\vartheta}, Q) d z, r_{p s}=r_{p s}(\sqrt{\vartheta}, Q), r=r(z, \sqrt{\vartheta}, Q), \\
& g(z, \sqrt{\vartheta}, Q)=\frac{1}{\sqrt{A\left(r_{p s}\right)-A(r) C\left(r_{p s}\right) / C(r)}}-\frac{1}{\sqrt{q\left(r_{p s}\right)} z} .
\end{aligned}
$$

For a certain charge $Q=Q_{0}, b_{R}=2 \int_{0}^{1} g(z, \sqrt{\vartheta}) d z$. In general, the coefficient $b_{R}$ can not be calculated analytically and, in this case it cannot be evaluated numerically. Here we expand it in powers of $\sqrt{\vartheta}$ as

$$
\begin{aligned}
& b_{R}=I_{R, 0}+I_{R, 1} \sqrt{\vartheta}+\frac{1}{2} I_{R, 2} \sqrt{\vartheta}^{2}+\cdots \\
& I_{R, 0}=\left.2 \int_{0}^{1} g(z, \sqrt{\vartheta})\right|_{\sqrt{\vartheta} \rightarrow 0} d z, I_{R, 1}=\left.2 \int_{0}^{1} \frac{d g}{d \sqrt{\vartheta}}\right|_{\sqrt{\vartheta} \rightarrow 0} d z, \cdots
\end{aligned}
$$

Because the values of various low derivative of $g(z, \sqrt{\vartheta})$ at $\vartheta \rightarrow 0$ is zero, we can get

$$
b_{R}=I_{R, 0}+O(\sqrt{\vartheta}) .
$$

Then, we can obtain the $\bar{a}, \bar{b}$ and $u_{p s}$, and describe them in Fig. 11.

Figure 1 tells us that given a certain charge, with the increases of $\sqrt{\vartheta}$ the coefficient $\bar{a}$ increases, the $\bar{b}$ and the minimum impact parameter $u_{p s}$ decrease. It also shows that: i) when $\sqrt{\vartheta}$ is strong, $Q_{\max }$ is small, so that the coefficients $\bar{a}, \bar{b}$ and $u_{p s}$ are close to those of the noncommutative Schwarzschild black hole; ii) when $\sqrt{\vartheta}$ is weak, $Q_{\max }$ is big, so that the coefficients $\bar{a}, \bar{b}$ and $u_{p s}$ are close to those of the commutative Reissner-Nordström black hole; iii) when $\sqrt{\vartheta}$ is weak, the 

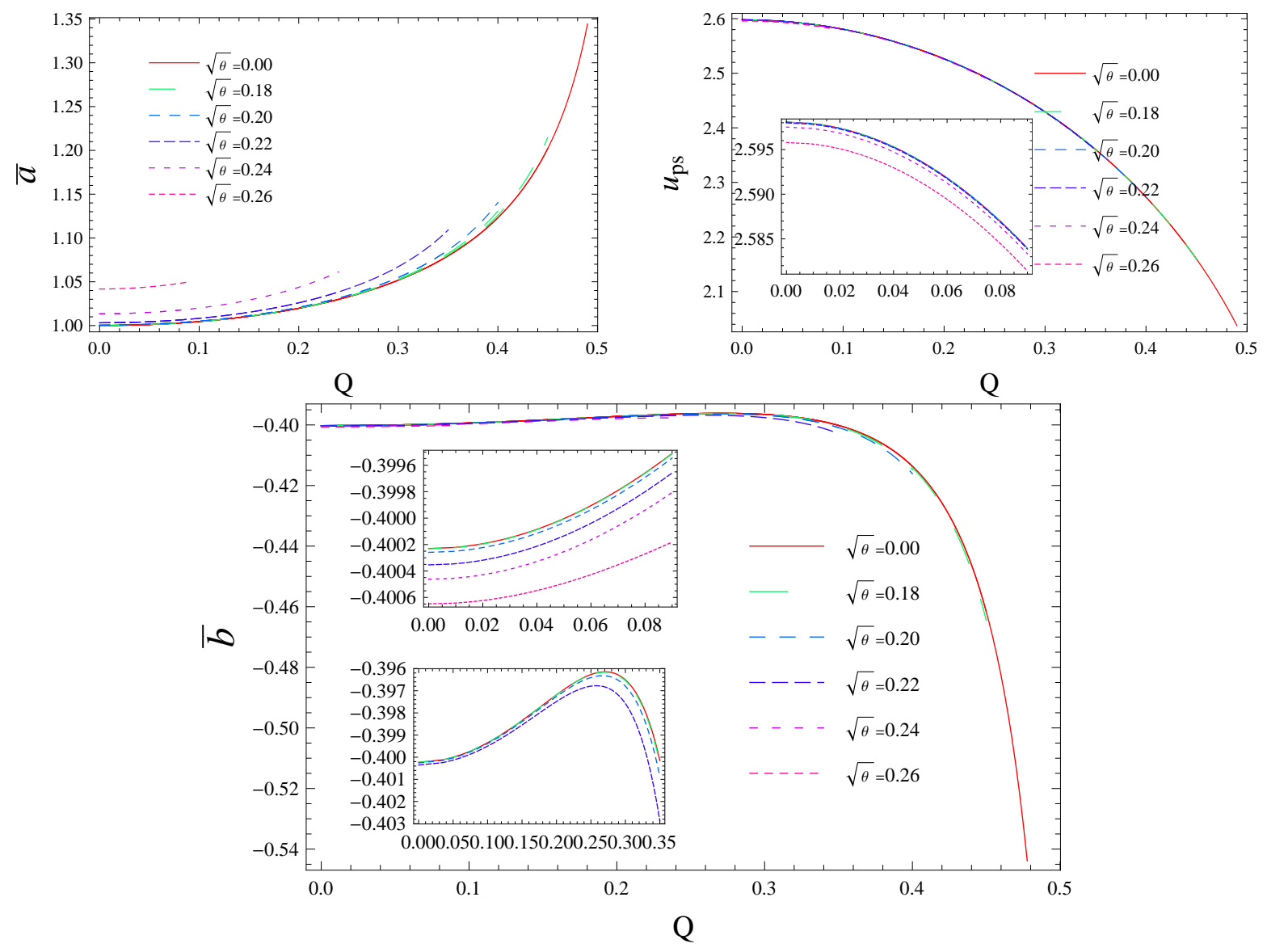

Figure 1: Variation of the coefficients of the strong field limit $\bar{a}, \bar{b}$ and the minimum impact parameter $u_{p s}$ with the spacetime noncommutative parameter $\sqrt{\vartheta}$ in the noncommutative Reissner-Nordström black hole spacetime.

effect of spacetime noncommutativity is obvious till that the charge quantity is very big; iv) the influence of the parameter $\sqrt{\vartheta}$ on $\bar{a}$ and $\bar{b}$ is bigger than that on $u_{p s}$. In principle we can distinguish a noncommutative Reissner-Nordström black hole from the commutative one and the noncommutative Schwarzschild black hole, and then maybe probe the value of the spacetime noncommutative constant by using strong field gravitational lensing.

Figure 2 shows the deflection angle $\alpha(\theta)$ evaluated at $u=u_{p s}+0.00326$. At this particular value of the impact parameter $\alpha(\theta)=2 \pi$ when $Q=0, \sqrt{\vartheta} \rightarrow 0$, which shows the photon just takes one loop around the black hole and the relativistic images begin to appear. Figure 2 indicates that the presence of $\sqrt{\vartheta}$ increases the deflection angle $\alpha(\theta)$ for the light propagated in the noncommutative Reissner-Nordström black hole spacetime. Comparing with those in the commutative one, we could extract the information about the size of spacetime noncommutative parameter $\sqrt{\vartheta}$ by using strong field gravitational lensing.

Considering the source, lens and observer are highly aligned, the lens equation in strong gravitational lensing can be written as 55

$$
\beta=\theta-\frac{D_{L S}}{D_{O S}} \Delta \alpha_{n},
$$




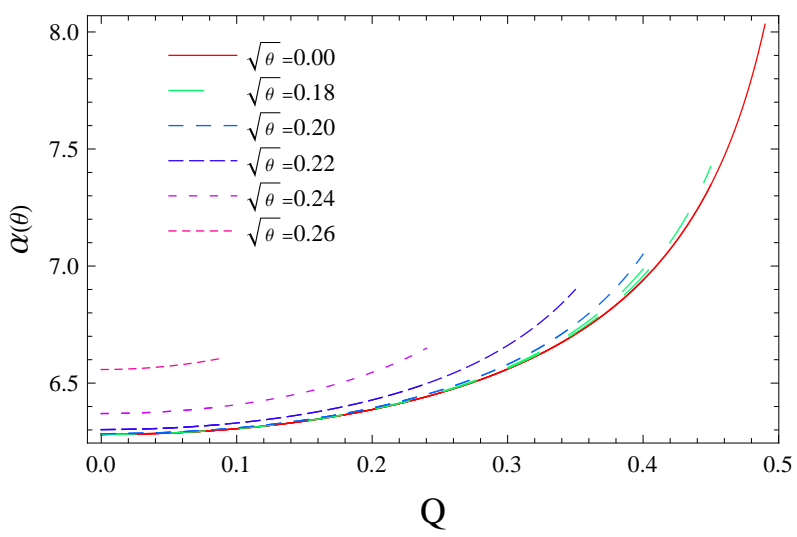

Figure 2: Deflection angles in the noncommutative Reissner-Nordström black hole spacetime evaluated at $u=u_{p s}+0.00326$ as functions of $Q$.

where $D_{L S}$ is the distance between the lens and the source, $D_{O S}=D_{L S}+D_{O L}, \beta$ is the angular separation between the source and the lens, $\theta$ is the angular separation between the image and the lens, $\Delta \alpha_{n}=\alpha-2 n \pi$ is the offset of deflection angle and $n$ is an integer. The position of the $n$-th relativistic image can be approximated as

$$
\theta_{n}=\theta_{n}^{0}+\frac{u_{p s} e_{n}\left(\beta-\theta_{n}^{0}\right) D_{O S}}{\bar{a} D_{L S} D_{O L}}
$$

where

$$
e_{n}=e^{\frac{\bar{b}-2 n \pi}{\bar{a}}}
$$

$\theta_{n}^{0}$ are the image positions corresponding to $\alpha=2 n \pi$. The magnification of the $n$-th relativistic image is given by

$$
\mu_{n}=\frac{u_{p s}^{2} e_{n}\left(1+e_{n}\right) D_{O S}}{\bar{a} \beta D_{L S} D_{O L}^{2}} .
$$

If $\theta_{\infty}$ represents the asymptotic position of a set of images in the limit $n \rightarrow \infty$, the minimum impact parameter $u_{p s}$ can be simply obtained as

$$
u_{p s}=D_{O L} \theta_{\infty}
$$

In the simplest situation, we consider only that the outermost image $\theta_{1}$ is resolved as a single image and all the remaining ones are packed together at $\theta_{\infty}$. Then the angular separation between the first image and other ones can be expressed as

$$
s=\theta_{1}-\theta_{\infty},
$$

and the ratio of the flux from the first image and those from the all other images is given by

$$
\mathcal{R}=\frac{\mu_{1}}{\sum_{n=2}^{\infty} \mu_{n}} .
$$


For a highly aligned source, lens and observer geometry, these observables can be simplified as

$$
\begin{aligned}
s & =\theta_{\infty} e^{\frac{\bar{b}-2 \pi}{\bar{a}}}, \\
\mathcal{R} & =e^{\frac{2 \pi}{\bar{a}}} .
\end{aligned}
$$

The strong deflection limit coefficients $\bar{a}, \bar{b}$, and the minimum impact parameter $u_{p s}$ can be obtained through measuring $s, \mathcal{R}$, and $\theta_{\infty}$. Then, comparing their values with those predicted by the theoretical models, we can identify the nature of the black hole lens.

\section{Numerical estimation of observational gravitational lensing parameters}

In this section, supposing that the gravitational field of the supermassive black hole at the Galactic center of the Milky Way can be described by the noncommutative Reissner-Nordström black hole metric, we estimate the numerical values for the coefficients and observables of the strong gravitational lensing; then we study the effect of the spacetime noncommutative parameter $\sqrt{\vartheta}$ on the gravitational lensing.

The mass of the central object of our Galaxy is estimated to be $2.8 \times 10^{6} M_{\odot}$ and its distance is around $8.5 \mathrm{kpc}$ [56]. For different $\vartheta$, the numerical value of the minimum impact parameter $u_{p s}$, the angular position of the asymptotic relativistic images $\theta_{\infty}$, the angular separation $s$, and the relative magnification of the outermost relativistic image with the other relativistic images $r_{m}$ are listed in Table 3 and 1 , and described in Fig. 3 .

Table 3: Numerical estimation for main observables and the strong field limit coefficients for the black hole at the center of our Galaxy, which is supposed to be described by the noncommutative Reissner-Nordström black hole with the charge $Q=0.09$. $R_{s}$ is Schwarzschild radius. $r_{m}=2.5 \log \mathcal{R}$.

\begin{tabular}{|c|c|c|c|c|c|c|}
\hline \hline$\sqrt{\vartheta}$ & $\theta_{\infty}(\mu$ arcsecs $)$ & $s$ ( $\mu$ arcsecs $)$ & $r_{m}$ (magnitudes) & $u_{p s} / R_{S}$ & $\bar{a}$ & $\bar{b}$ \\
\hline 0 & 16.7782 & 0.02153 & 6.79692 & 2.58396 & 1.00367 & -0.399510 \\
\hline 0.16 & 16.7782 & 0.02153 & 6.79691 & 2.58396 & 1.00367 & -0.399510 \\
\hline 0.18 & 16.7782 & 0.02154 & 6.79669 & 2.58396 & 1.00371 & -0.399514 \\
\hline 0.20 & 16.7781 & 0.02161 & 6.79342 & 2.58395 & 1.00419 & -0.399546 \\
\hline 0.22 & 16.7775 & 0.02205 & 6.77263 & 2.58385 & 1.00727 & -0.399658 \\
\hline 0.24 & 16.7741 & 0.02375 & 6.69627 & 2.58332 & 1.01876 & -0.399806 \\
\hline 0.26 & 16.7616 & 0.02883 & 6.49748 & 2.58140 & 1.04993 & -0.400182 \\
\hline \hline
\end{tabular}

From Table 3 and 1 , it is easy to see that our results reduce those in the commutative ReissnerNordström black hole sacetime [53] to $\sqrt{\vartheta} \rightarrow 0$. Table 3 shows that if $\sqrt{\vartheta}$ is strong, for example, $\sqrt{\vartheta}=0.26$, the observables $\theta_{\infty}, r_{m}$ and $s$ are close to those of the noncommutative Schwarzschild black hole [28]. Moreover, we also find that as the parameter $\sqrt{\vartheta}$ increases, the minimum impact parameter $u_{p s}$, the angular position of the relativistic images $\theta_{\infty}$, and the relative magnitudes $r_{m}$ decrease, but the angular separation $s$ increases. From Fig. 3, we find that the influence of the parameter $\sqrt{\vartheta}$ on $r_{m}$ and $s$ is bigger than that on $\theta_{\infty}$. This means that the bending angle is bigger and the relative magnification of the outermost relativistic image with the other relativistic images is smaller in the noncommutative Reissner-Nordström black hole spacetime. 
Table 4: Numerical estimation for main observables and the strong field limit coefficients for the black hole at the center of our Galaxy, which is supposed to be described by the noncommutative Reissner-Nordström black hole with the charge $Q=0.49$. $R_{s}$ is Schwarzschild radius. $r_{m}=2.5 \log \mathcal{R}$.

\begin{tabular}{|c|c|c|c|c|c|c|}
\hline \hline$\sqrt{\vartheta}$ & $\theta_{\infty}(\mu$ arcsecs $)$ & $s$ ( $\mu$ arcsecs $)$ & $r_{m}$ (magnitudes) & $u_{p s} / R_{S}$ & $\bar{a}$ & $\bar{b}$ \\
\hline 0 & 13.2321 & 0.0777923 & 5.07464 & 2.03784 & 1.34431 & -0.621671 \\
\hline 0.09 & 13.2321 & 0.0777923 & 5.07464 & 2.03784 & 1.34431 & -0.621671 \\
\hline 0.10 & 13.2321 & 0.0777923 & 5.07464 & 2.03784 & 1.34431 & -0.621671 \\
\hline 0.11 & 13.2321 & 0.0777923 & 5.07464 & 2.03784 & 1.34431 & -0.621671 \\
\hline 0.12 & 13.2321 & 0.0777939 & 5.07461 & 2.03784 & 1.34432 & -0.621673 \\
\hline 0.13 & 13.2321 & 0.0778157 & 5.07431 & 2.03783 & 1.34440 & -0.621707 \\
\hline 0.14 & 13.2321 & 0.0779688 & 5.07220 & 2.03783 & 1.34495 & -0.621932 \\
\hline \hline
\end{tabular}
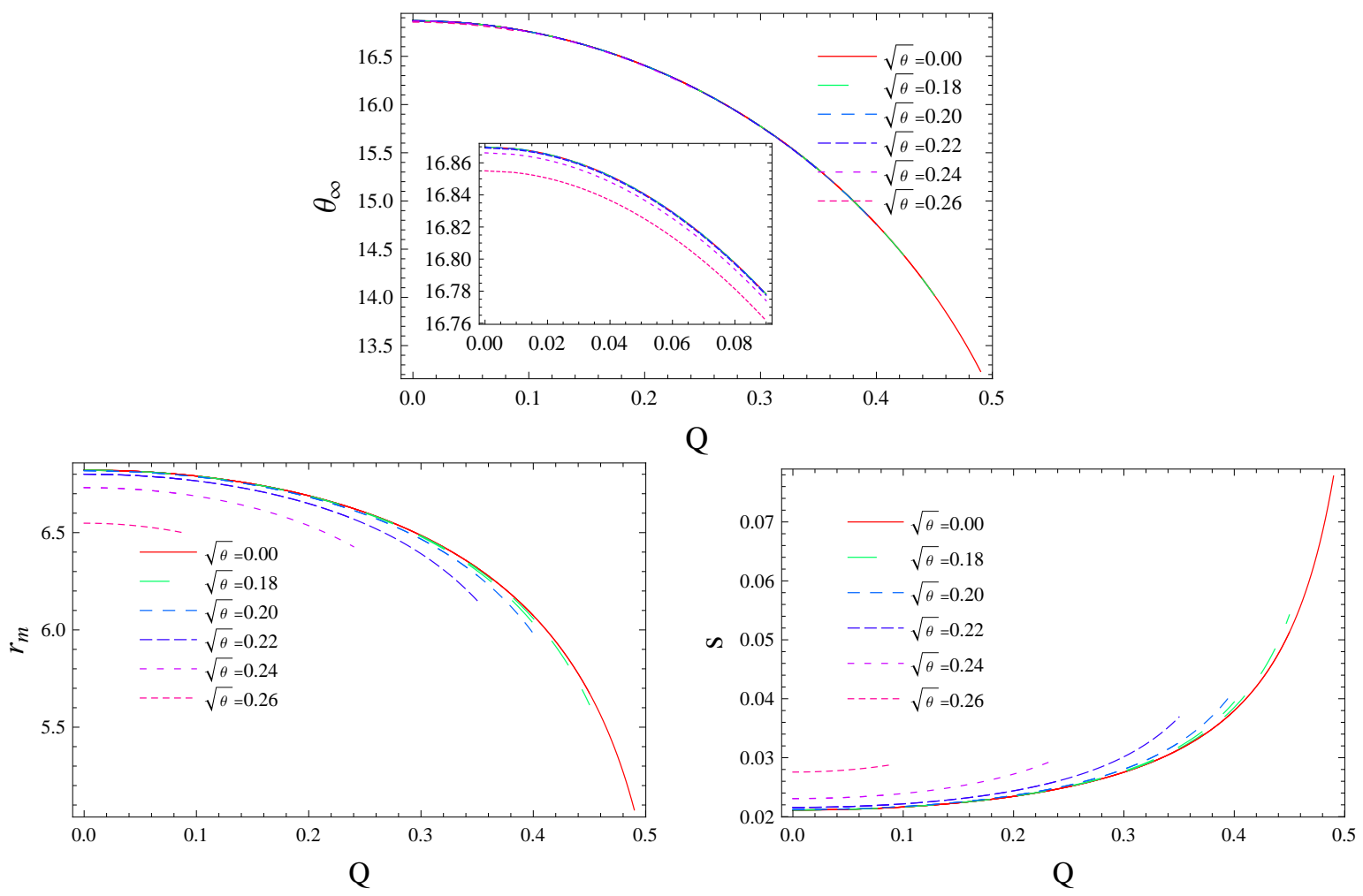

Figure 3: Strong gravitational lensing by the Galactic center black hole. Variation of the values of the angular position $\theta_{\infty}$, the relative magnitudes $r_{m}$, and the angular separation $s$ with parameter $Q$ in the noncommutative Reissner-Nordström black hole spacetime.

From Tab. 4 and Fig. 3 we also find that if $\sqrt{\vartheta}$ is weak, e.g. $0.12 \leq \sqrt{\vartheta} \leq 0.14$, the effect of spacetime noncommutativity is obvious till that the charge quantity is very big, e.g., $Q=0.49$. Tab. 1 also shows that the detectable lower limit of $\sqrt{\vartheta}$ is 0.12 . From Tab. 1, we can let the charge even bigger, i.e. $0.49<Q<0.5$, then the detectable lower limit lies between 0.11 and 0.12 . But it is only a numerical result, so the detectable lower limit 0.12 is more appropriate. Therefore we can 
conclude that the detectable scope of $\vartheta$ in a noncommutative Reissner-Nordström black hole lensing is $0.12 \leq \sqrt{\vartheta} \leq 0.26$.

In order to identify the nature of these three compact objects lensing, it is necessary for us to measure angular separation $s$ and the relative magnification $r_{m}$ in the astronomical observations. Table 1 tells us that the resolution of the extremely faint image is $\sim 0.07 \mu$ arc sec, which is too small. However, with the development of technology, the effects of the spacetime noncommutative constant $\sqrt{\vartheta}$ on gravitational lensing may be detected in the future.

\section{Summary}

Spacetime noncommutative constant would be a new fundamental natural constant which can affect the classical gravitational effect such as gravitational lensing. Studying the strong gravitational lensing can help us to probe the spacetime noncommutative constant. In this paper we have investigated strong field lensing in the noncommutative Reissner-Nordström black hole spacetime to study the influence of the spacetime noncommutative parameter on the strong gravitational lensing. The model was applied to the supermassive black hole in the Galactic center. Our results show that with the increase of the parameter $\sqrt{\vartheta}$, the minimum impact parameter $u_{p s}$, the angular position of the relativistic images $\theta_{\infty}$ and the relative magnitudes $r_{m}$ decrease, and the angular separation $s$ increases.

Our results also show that i) if $\sqrt{\vartheta}$ is strong, $Q_{\max }$ is small, so that the observables $\theta_{\infty}, r_{m}$ and $s$ are close to those of the noncommutative Schwarzschild black hole lensing; ii) if $\sqrt{\vartheta}$ is weak, $Q_{\max }$ is big, so that the observables $\theta_{\infty}, r_{m}$ and $s$ are close to those of the commutative ReissnerNordström black hole lensing; iii) if $\sqrt{\vartheta}$ is weak, e.g. $0.12 \leq \sqrt{\vartheta} \leq 0.14$, the effect of spacetime noncommutativity is obvious till that the charge quantity is very big, and if $\sqrt{\vartheta}<0.12$, we cannot probe the $\vartheta$ at all; iv) the influence of the parameter $\sqrt{\vartheta}$ on $r_{m}$ and $s$ is bigger than that on $\theta_{\infty}$.

In short, the detectable scope of $\vartheta$ in a noncommutative Reissner-Nordström black hole lensing is $0.12 \leq \sqrt{\vartheta} \leq 0.26$, which is wider than that in a noncommutative Schwarzschild black hole lensing, $0.18 \leq \sqrt{\vartheta} \leq 0.26$ [28]. This may offer a way to distinguish a noncommutative Reissner-Nordström black hole from the commutative one and the noncommutative Schwarzschild black hole and probe the spacetime noncommutative constant with the astronomical instruments in the future.

\section{Acknowledgments}

This work was partially supported by Hunan Provincial Natural Science Foundation of China under Grant No. 11JJ3014 and the Scientific Research Foundation for the introduced talents of Hunan Institute of Humanities, Science and Technology. J. Jing's work was supported by the NNSFC No.10875040 and No.10935013, 973 Program No. 2010CB833004 and PCSIRT under Grant No. IRT0964.

\section{References}

[1] R. Garattini and P. Nicolini, Noncommutative approach to the cosmological constant problem, Phys. Rev. D 83064021 (2011). 
[2] N. Seiberg and E. Witten, String Theory and Noncommutative Geometry, J. High Energy Phys. 09 (1999) 032; E. Witten, Bound States Of Strings And p-Branes, Nucl. Phys. B 460335 (1996).

[3] J. Bellissard, A. van Elst and H. Schulz-Baldes, The Non-Commutative Geometry of the Quantum Hall Effect, J. Math. Phys. 355373 (1994).

[4] J. Gamboa et al., The Landau problem and noncommutative quantum mechanics, Mod. Phys. Lett. A 162075 (2001); J. Gamboa, M. Loewe and J. C. Rojas, Non-Commutative Quantum Mechanics, Phys. Rev. D 64067901 (2001); P. A. Horvathy, The non-commutative Landau problem, Ann. Phys. 299128 (2002).

[5] M. Marcolli and E. Pierpaoli, Early universe models from noncommutative geometry, arXiv:0908.3683

[6] W. T. Kim and J. J. Oh, Noncommutative open strings from Dirac quantization, Mod. Phys. Lett. A 15 $1597(2000)$.

[7] R. Jackiw, Physical Instances of Noncommuting Coordinates, Nucl. Phys. Proc. Suppl. 10830 (2002); Phys. Part. Nucl. 33 S6 (2002); L. Lan-dau, Zh. Eksp. Teor. Fiz. 11592 (1941), [English trans-lation: J. Phys. USSR, 571 (1941)].

[8] S. Deser, R. Jackiw and S. Templeton, Topologically massive gauge theories, Ann. Phys. 140372 (1982), [Erratum ibid. 185406 (1988)] [Ann. Phys. (NY) 281 (2000) 409].

[9] H. S. Snyder, Quantized space-time, Phys. Rev. 7138 (1947); The Electromagnetic field in Quantized Space-Time, Phys. Rev. 72 68, (1947).

[10] C. N. Yang, On quantized space-time, Phys. Rev. 72 874, (1947).

[11] A. Connes, Noncommutative Geometry, Academic Press, New York, (1994).

[12] A. Connes and M. Marcolli, A walk in the noncommutative garden, math.QA/0601054.

[13] E. Akofor, Quantum Theory, Noncommutativity and Heuristics, Ph. D. Dissertation, arXiv:1012.5133.

[14] A. Smailagic and E. Spallucci, Feynman Path Integral on the Noncommutative Plane, J. Phys. A 36 L467 (2003), A. Smailagic and E. Spallucci, UV divergence-free QFT on noncommutative plane, J. Phys. A 36 L517 (2003),

[15] S. Ansoldi, P. Nicolini, A. Smailagic and E. Spallucci, Noncommutative geometry inspired charged black holes, Phys. Lett. B 645, 261 (2007).

[16] J. Gomis and T. Mehen, Space-Time Noncommutative Field Theories And Unitarity, Nucl. Phys. B 591 265 (2000); K. Morita, Y. Okumura and E. Umezawa, Lorentz Invariance And Unitarity Problem In Non-Commutative Field Theory, Prog. Theor. Phys. 110989 (2003); P. Fischer and V. Putz, No UV/IR Mixing in Unitary Space-Time Noncommutative Field Theory, Eur. Phys. J. C 32269 (2004); Y. Liao and K. Sibold, Time-ordered Perturbation Theory on Noncommutative Spacetime II: Unitarity, Eur. Phys. J. C 25479 (2002); T. Ohl R. Rückl and J. Zeiner, Unitarity of Time-Like Noncommutative Gauge Theories: The Violation of Ward Identities in Time-Ordered Perturbation Theory, Nucl. Phys. B 676229 (2004). 
[17] The notation $\vartheta$ used here is a constant as well as Plank constant $\hbar$, but we still call it a spacetime noncommutative parameter since it up to now is undetermined.

[18] A. Smailagic, E. Spallucci, Lorentz invariance, unitarity and UV-finiteness of QFT on noncommutative spacetime, J. Phys. A 377169 (2004).

[19] P. Nicolini, Noncommutative Black Holes, The Final Appeal To Quantum Gravity: A Review, Int. J. Mod. Phys. A 24, 1229 (2009); S. Ansoldi, P. Nicolini, A. Smailagic and E. Spallucci, Noncommutative geometry inspired charged black holes Phys. Lett. B 645, 261 (2007); P. Nicolini and E. Spallucci, Noncommutative geometry inspired wormholes and dirty black holes, Class. Quant. Grav. 27015010 (2010); A. Smailagic and E. Spallucci, 'Kerrr' black hole: the Lord of the String, Phys. Lett. B 688, 82 (2010); L. Modesto and P. Nicolini, Charged rotating noncommutative black holes, Phys. Rev. D 82, 104035 (2010); E. Spallucci, A. Smailagic and P. Nicolini, Non-commutative geometry inspired higher-dimensional charged, black holes, Phys. Lett. B 670, 449 (2009).

[20] K. Nozari and S. H. Mehdipour, Hawking Radiation as Quantum Tunneling from Noncommutative Schwarzschild Black Hole, Class. Quant. Grav. 25 175015, (2008); W. Kim, E. J. Son and M. Yoon, Thermodynamic similarity between the noncommutative Schwarzschild black hole and the Reissner-Nordström black hole, JHEP 0804 (2008) 042; B. Vakili, N. Khosravi and H. R. Sepangi, Thermodynamics of noncommutative de Sitter spacetime, Int. J. Mod. Phys. D 18 159, (2009); M. Buric and J. Madore, Spherically Symmetric Noncommutative Space: $d=4$, Eur. Phys. J. C 58 347, (2008); W. H. Huang and K. W. Huang, Thermodynamics on Noncommutative Geometry in Coherent State Formalism, Phys. Lett. B 670 416, (2009); M. Park, Smeared Hairs and Black Holes in Three-Dimensional de Sitter Spacetime, Phys. Rev. D 80 084026, (2009); K. Nozari and S. H. Mehdipour, Parikh-Wilczek Tunneling from Noncommutative Higher Dimensional Black Holes, JHEP 0903 (2009) 061; J. J. Oh and C. Park, Gravitational Collapse of the Shells with the Smeared Gravitational Source in Noncommutative Geometry, JHEP 1003:086 (2010); I. Arraut, D. Batic and M. Nowakowski, Maximal extension of the Schwarzschild spacetime inspired by noncommutative geometry, arXiv:1001.2226[gr-qc].

[21] H. Garcia-Compean and C. Soto-Campos, Noncommutative Effects in the Black Hole Evaporation in Two Dimensions, Phys. Rev. D 74 104028, (2006); E. D. Grezia, G. Esposito and G. Miele, Gravitational amplitudes in black-hole evaporation: the effect of non-commutative geometry, Class. Quant. Grav. 23 6425, (2006); E. D. Grezia, G. Esposito and G. Miele, Black hole evaporation in a spherically symmetric non-commutative space-time, J. Phys. A 41 164063, (2008); Y. S. Myung, Y. W. Kim and Y. J. Park, Thermodynamics and evaporation of the noncommutative black hole, JHEP 0702 (2007) 012; R. Casadio and P. Nicolini, The decay-time of non-commutative micro-black holes, JHEP $0811(2008) 072$.

[22] Y.-G. Miao, Z. Xue and S.-J. Zhang, Quantum tunneling and spectroscopy of noncommutative Kerr black hole, arXiv:1102.0074v2. 
[23] S. W. Wei, Y. X. Liu, Z. H. Zhao and C.-E Fu, Area spectrum of Schwarzschild black hole inspired by noncommutative geometry, arXiv:1004.2005v2 [hep-th];

[24] R. B. Mann and P. Nicolini, Cosmological production of noncommutative black holes, arXiv:1102.5096.

[25] J. W. Moffat, Ultraviolet Complete Quantum Gravity, Eur. Phys. J. Plus 12643 (2011).

[26] D. M. Gingrich, Noncommutative geometry inspired black holes in higher dimensions at the LHC, JHEP 1105:022 (2010); M. Bleicher and P. Nicolini, Large extra dimensions and small black holes at the LHC, J. Phys. Conf. Ser. 237, 012008 (2010).

[27] O. Bertolami and C. A. D. Zarro, Towards a Noncommutative Astrophysics, Phys. Rev. D 81025005 (2010).

[28] C. Ding, S. Kang, C.-Y. Chen, S. Chen and J. Jing, Strong gravitational lensing in a noncommutative black-hole spacetime, Phys. Rev. D 83084005 (2011).

[29] J. Wambsganss, Gravitational lensing in astronomy, Liv. Rev. Rel. 1 (1998); P. Schneider, J. Ehlers and E. E. Falco, Gravitational Lenses, (Berlin: Springer 1992); H. Hoekstra and B. Jain, Weak Gravitational Lensing and its cosmological applications, Ann. Rev. Nuc. Particle Sci. 5899 (2008).

[30] C. Darwin, Proc. R. Soc. London, 249, 180 (1959).

[31] K. S. Virbhadra, D. Narasimha and S. M. Chitre, Role of the scalar field in gravitational lensing, Astron. Astrophys. 337, 1-8 (1998).

[32] K. S. Virbhadra and G. F. R. Ellis, Schwarzschild black hole lensing, Phys. Rev. D 62, 084003 (2000).

[33] C. M. Claudel, K. S. Virbhadra and G. F. R. Ellis, The geometry of photon surfaces, J. Math. Phys. 42, 818 (2001).

[34] K. S. Virbhadra and G. F. R. Ellis, Gravitational Lensing By Naked Singularities, Phys. Rev.D 65, 103004 (2002).

[35] S. Frittelly, T. P. Kling and E. T. Newman, Spacetime perspective of Schwarzschild lensing, Phys. Rev. D 61, 064021 (2000).

[36] A. Bhadra, Gravitational lensing by a charged black hole of string theory, Phys. Rev. D 67, 103009 (2003).

[37] K. Sarkar and A. Bhadra, Strong field gravitational lensing in scalar tensor theories, Class. Quant. Grav. 23, 6101 (2006).

[38] E. F. Eiroa, G. E. Romero and D. F. Torres, Reissner-Nordstrom black hole lensing, Phys. Rev. D 66, 024010 (2002).

[39] R. A. Konoplya, Particle motion around magnetized black holes: Preston-Poisson space-time, Phys. Rev. D 74, 124015 (2006); Magnetized black hole as a gravitational lens, Phys. Lett. B 644, 219 (2007).

[40] N. Mukherjee and A. S. Majumdar, Particle motion and gravitational lensing in the metric of a dilaton black hole in a de Sitter universe, Gen. Rel. Grav. 39583 (2007). 
[41] V. Perlick, On the exact gravitational lens equation in spherically symmetric and static spacetimes, Phys. Rev. D 69, 064017 (2004).

[42] A. Y. Bin-Nun, Strong Gravitational Lensing by Sgr A*, Class. Quant. Grav. 28, 114003 (2011).

[43] S.-W. Wei, Y.-X. Liu, C.-E Fu and K. Yang, Strong field limit analysis of gravitational lensing in Kerr-Taub-NUT spacetime, arXiv:1104.0776.

[44] A. S. Majumdar and N. Mukherjee, Braneworld black holes in cosmology and astrophysics, Int. J. Mod. Phys. D 141095 (2005); R. Whisker, Strong gravitational lensing by braneworld black holes, Phys. Rev. D 71064004 (2005).

[45] A. S. Eddington, Internal Constitution of the Stars (Cambridge University Press, 1926); N. K. Glendenning, Compact Stars (A\&A Library, Springer-Verlag New York Inc., 2000), p. 82.

[46] J. Bally and E. R. Harrison, The electrically polarized universe, Astrophys. J. 220743 (1978); E. Olson and M. Bailyn, Internal Structure Of Multicomponent Static Spherical Gravitating Fluids, Phys. Rev. D 12, 3030 (1975); E. Olson and M. Bailyn, Charge Effects In A Static, Spherically Symmetric, Gravitating Fluid, Phys. Rev. D 132204 (1976).

[47] C. R. Ghezzi, Phys. Rev. D 72104017 (2005); C. R. Ghezzi and P. S. Letelier, Numeric simulation of relativistic stellar core collapse and the formation of Reissner-Nordstrom black holes, Phys. Rev. D. 75 024020 (2007).

[48] A. N. Baushev and P. Chardonnet, Electric charge estimation of a new-born black hole, Int. J. Mod. Phys. D 182035 (2009); R. Ruffini, J. D. Salmonson, J. R. Wilson and S.-S. Xue, On the pair electromagnetic pulse of a black hole with electromagnetic structure, Astron. Astrophys. 350334 (1999); J. A. de Diego, D. Dultzin-Hacyan, J. G. Trejo and D. Nún̈ez, astro-ph/0405237.

[49] H. J. M. Cuesta, A. P.-Firme and A. P.- Lorenzana, Charge asymmetry in the brane world and the formation of charged black holes, Phys. Rev. D 67, 087702 (2003).

[50] S. L. Shapiro and S. A. Teukolsky, White Dwarfs, Black Holes and Neutron Stars: The Physics of Compact Objects (Wiley, New York, 1983).

[51] R. Ruffini et al., On a possible GRB-supernova time sequence, Astrophys. J. 555 L107 (2001).

[52] H. J. M. Cuesta, A. P.-Firme and A. P.-Lorenzana, Quasi-Equatorial Gravitational Lensing by Spinning Black Holes in the Strong Field Limit, Phys. Rev. D. 67103006 (2003).

[53] V. Bozza, Gravitational lensing in the strong field limit, Phys. Rev. D 66, 103001 (2002).

[54] S. Chen and J. Jing, Strong field gravitational lensing in the deformed Hrava-Lifshitz black hole, Phys. Rev. D 80, 024036 (2009).

[55] V. Bozza, S. Capozziello, G. lovane and G. Scarpetta, Strong field limit of black hole gravitational lensing, Gen. Rel. Grav. 33, 1535 (2001). 
[56] D. Richstone et al., Supermassive Black Holes and the Evolution of Galaxies, Nature 395 A14 (1998); F. Melia and H. Falcke, The Supermassive Black Hole at the Galactic Center, Ann. Rev. Astron. Astrophys. 39309 (2001). 